Swarthmore College

Works

Physics \& Astronomy Faculty Works

Physics \& Astronomy

2-20-2003

\title{
Protoplanetary Disk Mass Distribution In Young Binaries
}

Eric L.N. Jensen

Swarthmore College, ejensen1@swarthmore.edu

R. L. Akeson

Follow this and additional works at: https://works.swarthmore.edu/fac-physics

Part of the Astrophysics and Astronomy Commons

Let us know how access to these works benefits you

\section{Recommended Citation}

Eric L.N. Jensen and R. L. Akeson. (2003). "Protoplanetary Disk Mass Distribution In Young Binaries". Astrophysical Journal. Volume 584, Issue 2. 875-881. DOI: 10.1086/345719

https://works.swarthmore.edu/fac-physics/2

This work is brought to you for free by Swarthmore College Libraries' Works. It has been accepted for inclusion in Physics \& Astronomy Faculty Works by an authorized administrator of Works. For more information, please contact myworks@swarthmore.edu. 


\title{
PROTOPLANETARY DISK MASS DISTRIBUTION IN YOUNG BINARIES
}

\author{
ERIC L. N. JENSEN \\ Department of Physics and Astronomy, Swarthmore College, Swarthmore, PA 19081; ejensen1@swarthmore.edu \\ AND \\ RACHEL L. AKESON \\ Interferometry Science Center, California Institute of Technology, MS 100-22, 1201 East California Boulevard, Pasadena, CA 91125; \\ rla@ipac.caltech.edu \\ Received 2002 September 4; accepted 2002 October 22
}

\begin{abstract}
We present millimeter-wave continuum images of four wide (separations 210-800 AU), young stellar binary systems in the Taurus-Auriga star-forming region. For all four sources, the resolution of our observations is sufficient to determine the millimeter emission from each of the components. In all four systems, the primary star's disk has stronger millimeter emission than the secondary's, and in three of the four the secondary is undetected; this is consistent with predictions of recent models of binary formation by fragmentation. The primaries' circumstellar disk masses inferred from these observations are comparable to those found for young single stars, confirming that the presence of a wide binary companion does not prevent the formation of a protoplanetary disk. Some of the secondaries show signatures of accretion ( $\mathrm{H} \alpha$ emission and $K-L$ excesses), yet their millimeter fluxes suggest that very little disk mass is present.
\end{abstract}

Subject headings: binaries: general — circumstellar matter — planetary systems: formation planetary systems: protoplanetary disks — stars: formation — stars: pre-main-sequence

On-line material: color figures

\section{INTRODUCTION}

The majority of stars are in binary or multiple systems during both the pre-main-sequence and main-sequence phases of stellar evolution (see, e.g., reviews by Mathieu 1994 and Mathieu et al. 2000). Therefore, understanding the causes and effects of multiplicity is an essential ingredient of complete models of both star and planet formation. Circumstellar disks play a crucial role in both processes, by providing conduits for material to accrete onto the stars and by providing sites for planet formation.

Only in the last few years have observations of young binaries and theories of binary formation both advanced to the point that direct comparison of observations and theory is possible. While such comparisons still cannot definitively establish the mechanism(s) of binary formation (see, e.g., Clarke 2001), increasingly there are opportunities to test predictions of binary formation models with observational data. Current observations are consistent with the formation of most low-mass binary systems by scale-free fragmentation of a molecular cloud (Clarke 2001; Ghez 2001). One notable prediction of such models is that the more massive star in the binary should always harbor the more massive circumstellar disk (Bate 2000).

We set out to test this prediction with the observations presented here. Previous work has shown that primary stars in young binary systems have more active (and thus perhaps more massive) disks than secondaries do. Primaries tend to have stronger signatures of the presence of circumstellar disks (red $K-L$ colors) and of accretion from such disks (strong $\mathrm{H} \alpha$ and $\mathrm{Br} \gamma$ emission) than secondaries do (Prato \& Simon 1997; Duchêne et al. 1999; White \& Ghez 2001; Prato \& Monin 2001). However, such observations reflect the disk conditions near the stellar surfaces, where accretion takes place and where the vast majority of the near-infrared flux is produced. These observations suggest a dependence of disk properties on stellar mass in binaries, but in general these diagnostics (especially $\mathrm{H} \alpha$ equivalent width) are at most only marginally correlated with disk mass (Beckwith et al. 1990, hereafter BSCG; Osterloh \& Beckwith 1995). Thus, the optical and near-IR observations do not directly address the question of total disk masses in these systems, making comparison with theoretical predictions problematic. A measurement of the disk masses with millimeter interferometry is necessary for direct comparison with theoretical predictions. The work we present here has the advantages that we (1) observed circumstellar disks at a wavelength $(\lambda=1.3$ $\mathrm{mm}$ ) at which they are likely to be optically thin, to probe their total masses; (2) resolved both components in the binaries; and (3) observed systems with millimeter fluxes that are typical of average $\mathrm{T}$ Tauri stars, not anomalously large.

A few binaries have been previously observed with millimeter interferometers, but the interpretation of their disk masses and morphologies is complicated by the presence of unresolved additional pairs in triple or quadruple systems (e.g., T Tau, Akeson, Koerner, \& Jensen 1998; UZ Tau, Jensen, Koerner \& Mathieu 1996a). Also, some of these observations have resolved the circumbinary disks, but not the binary itself (GG Tau, Koerner, Sargent, \& Beckwith 1993; Guilloteau, Dutrey, \& Simon 1999; UY Aur, Duvert et al. 1998). Thus, these observations do not reveal the distribution of disk mass between the two stars in each system. The observations we report here avoid the complication of unresolved additional components (as far as we know, except in the case of UX Tau B) and thus allow us to determine the disk mass distribution in the circumstellar disks of four young binary systems.

We present our observations in $\S 2$. We then discuss the disk properties implied by our observations and other data, 
TABLE 1

YOUNG BiNARIES OBSERVED

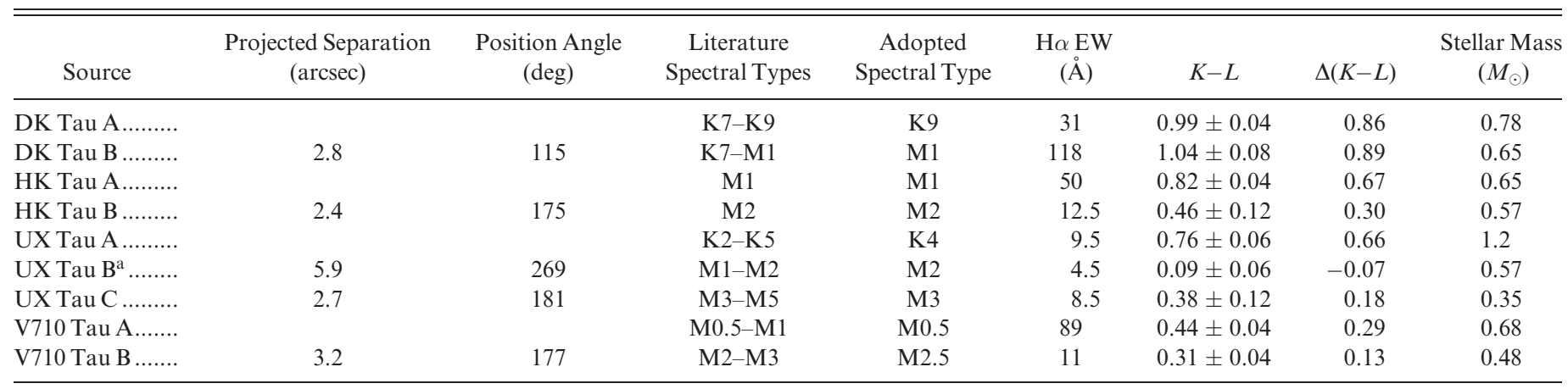

Notes.-Position angle and separation values are from Leinert et al. 1993 and are relative to the primary star. For V710 Tau, component A here is the optical primary, although component B (V710 Tau S) is brighter at $2.2 \mu \mathrm{m}$. Adopted spectral types and H $\alpha$ equivalent widths are from Hartigan, Strom, \& Strom 1994 for V710 Tau and Duchêne et al. 1999 for all other stars; $K-L$ values are from White \& Ghez 2001. Spectral types were used to derive effective temperatures based on Table 2 of Luhman 1999 for the M stars and on the dwarf temperature scale in Cox 2000 for the K stars. The masses were then estimated from the Baraffe et al. 1998 models assuming an age of 3 Myr, since Luhman 2000 found a sample of young stars in Taurus to lie between the 1 and 3 Myr tracks of the Baraffe et al. 1998 models. Although this method does not produce precise values for the individual masses, the relative masses should be relatively well determined.

a UX Tau B is a 0".14 binary (Duchêne 1999), unresolved in our data but visible in the HST data in Fig. 1.

in $\S 3$, and the implications of these results for our understanding of binary star formation and the prospects for planet formation in binary systems, in $\S 4$.

\section{OBSERVATIONS}

We selected our targets from among known pre-mainsequence binaries in Taurus-Auriga based on the following criteria: separation greater than 1 !"1, wide enough to resolve with the Owens Valley Millimeter Array (which has a resolution of 1 ". $1 \times 0$ ". 8 for a full track in the high-resolution configuration); and $\lambda=1.3 \mathrm{~mm}$ detection in the surveys of BSCG or Osterloh \& Beckwith (1995). We did not restrict our sample to the brightest $1.3 \mathrm{~mm}$ sources, as limited sensitivity has necessitated in the past. We avoided triple and quadruple systems in which the closer pair(s) would be unresolved in our Owens Valley Radio Observatory (OVRO) observations, since the presence of a companion with separation less than $100 \mathrm{AU}$ is well correlated with decreased millimeter emission (BSCG; Osterloh \& Beckwith 1995; Jensen, Mathieu, \& Fuller 1996b). We included UX Tau in our sample since the $\mathrm{A}, \mathrm{B}$, and $\mathrm{C}$ components can be resolved by OVRO; we did not realize until later that UX Tau B is itself a close binary. Our sample consists of three binary systems (DK Tau, HK Tau, and V710 Tau) and one quadruple system (UX Tau); source properties are summarized in Table 1. We note that the single-dish millimeter fluxes of these systems (35-63 mJy) are fairly typical of T Tauri star fluxes; the median flux among single stars in the samples of Osterloh \& Beckwith (1995) and BSCG (including nondetections) is 36 $\mathrm{mJy}$, while the median flux among binary stars is somewhat lower.

We observed the four systems in Table 1 with the Owens Valley Millimeter Array on 2002 January 11 and 19 and February 10. We obtained data in both the low-resolution array (baselines $36-115 \mathrm{~m}$, hour angle range $\pm 3^{\mathrm{h}}$ ) and the high-resolution array (baselines 35-240 $\mathrm{m}$, hour angle range $\left.0^{\mathrm{h}}-3^{\mathrm{h}}\right)$. The correlator was configured with two wideband channels centered around $230 \mathrm{GHz}$ and narrowband channels centered around ${ }^{12} \mathrm{CO}(2 \rightarrow 1)$. The combination of the two sidebands resulted in a total continuum bandwidth of $4 \mathrm{GHz}$.
During each observation, the array pointing was cycled among the four sources to provide similar hour angle coverage for each. The quasar J0449+135 was used as the gain calibrator, and 3C 454.3 and 3C 273 were observed for use as flux calibrators. As our observations contained no measurements of primary flux calibrators (e.g., planets), we estimate the absolute flux calibration uncertainty to be $20 \%$; relative fluxes are unaffected by this uncertainty. This uncertainty is based on the scatter in other measurements of $3 \mathrm{C}$ 454.3 and 3C 273 at OVRO that were calibrated using primary flux calibrators. The gain and flux calibrations were applied in the OVRO MMA package. Maps were constructed and CLEANed in the MIRIAD package using a robust weighting, which resulted in a 1 ." $5 \times 1$ "! 3 beam.

All four targets show at least one clearly detected millimeter continuum source (Table 2; Fig. 1). However, CO emission was not detected toward any of these sources; $3 \sigma$

TABLE 2

Millimeter FluXes

\begin{tabular}{cccc}
\hline \hline Source & $\begin{array}{c}1.3 \mathrm{~mm} \\
\text { OVRO Flux } \\
(\mathrm{mJy})\end{array}$ & $\begin{array}{c}\text { Source Size } \\
(\operatorname{arcsec})\end{array}$ & $\begin{array}{c}1.3 \mathrm{~mm} \\
\text { Single-Dish Flux } \\
(\mathrm{mJy})\end{array}$ \\
\hline DK Tau A....... & $18 \pm 2.2$ & Point & $35 \pm 7$ \\
DK Tau B ........ & $<6.6$ & $\ldots$ & $\ldots$ \\
HK Tau A........ & $16 \pm 2.1$ & Point & $41 \pm 5$ \\
HK Tau B ........ & $11 \pm 2.1$ & Point & $\ldots$ \\
UX Tau A ........ & $52 \pm 2.2$ & $1.1 \times 0.6$ & $63 \pm 10$ \\
UX Tau B ........ & $<6.6$ & $\ldots$ & $\ldots$ \\
UX Tau C ........ & $<6.6$ & $\ldots$ & $60 \pm 7$ \\
V710 Tau A...... & $33 \pm 2.2$ & Point & $\ldots$ \\
V710 Tau B ....... & $<6.6$ & $\ldots$ & $\ldots$ \\
\hline
\end{tabular}

a Errors given are the rms noise in the maps and do not include the absolute flux calibration uncertainty of $20 \%$. Upper limits given are $3 \sigma$.

b The size given for UX Tau A is for a Gaussian fit to the emission. In the fit to HK Tau, there is some diffuse residual emission between the two sources that is not included above. The total flux from HK Tan is $30 \pm 2.1$ $\mathrm{mJy}$.

${ }^{c}$ The single-dish fluxes are taken from Osterloh \& Beckwith 1995 or BSCG and have a beam size of $11^{\prime \prime}$, encompassing all the stars in each system. The uncertainties do not include the absolute flux calibration uncertainty. 

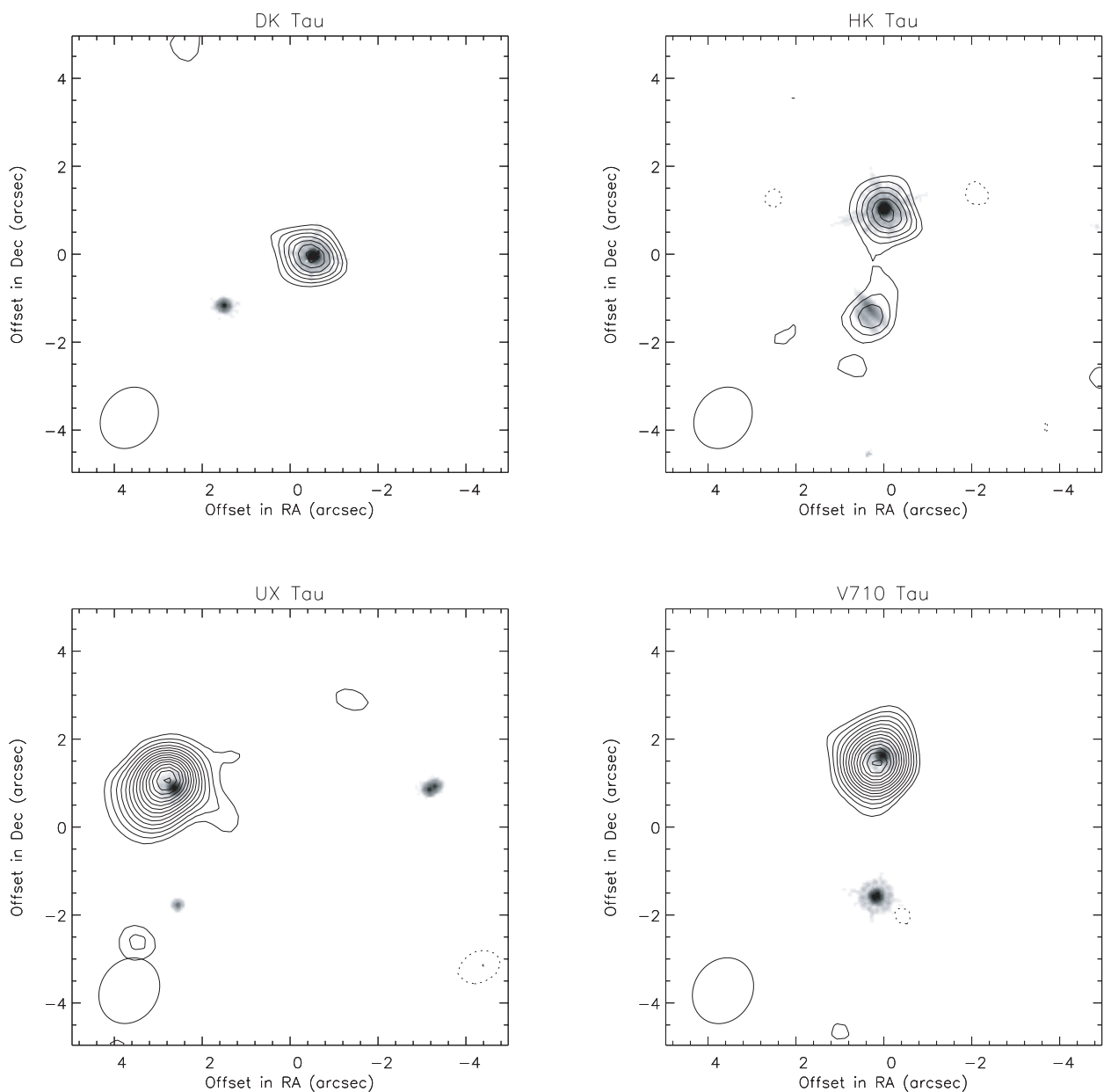

FIG. 1.-Millimeter emission from four young multiple systems. The contours show the $\lambda=1.3 \mathrm{~mm}$ flux observed with the Owens Valley Millimeter Array, overlaid on gray-scale optical images from archival HST data. In all cases the primary star dominates the disk emission from the system; only in HK Tau, the system with the mass ratio closest to 1 (see Table 1), is there detectable emission from the secondary. The contours are in steps of the rms noise in the millimeter maps, 2.1-2.2 mJy, starting at the $3 \sigma$ contour; negative contours (dashed lines) have the same steps, starting at $-3 \sigma$. The beam size (1".5 $\times 1$ "'.3) is shown at bottom left in each panel. [See the electronic edition of the Journal for a color version of this figure.]

limits are $0.7-0.8 \mathrm{Jy}, T_{\text {antenna }}=2.2-2.7 \mathrm{~K}$ at a resolution of $0.33 \mathrm{~km} \mathrm{~s}^{-1}$, or $0.3-0.4 \mathrm{Jy}, T_{\text {antenna }}=1.0-1.3 \mathrm{~K}$ when the spectra are binned to a resolution of $2 \mathrm{~km} \mathrm{~s}^{-1}$. As discussed at the end of this section, our observations are less sensitive to emission on scales greater than $5^{\prime \prime}$.

Three of the four systems show only one detected millimeter source. To determine which star is the source of the millimeter continuum emission, we overlaid the millimeter maps onto archival Hubble Space Telescope (HST) optical images of our sources taken in the F814W filter (similar to Cousins $I$ ). The absolute pointing accuracy of our observations is 0 .' 1 , as measured by quasar observations, but uncertainties in positions of the guide stars typically limit the accuracy of the HST/WFPC2 coordinates to $0.7 \mathrm{rms}$ (Baggett et al. 2002, § 5.4). Thus, to set the absolute coordinates in the HST images more accurately we used coordinates for our targets from the Hipparcos catalog (for UX Tau only) or from the USNO-A2.0 catalog (Monet et al. 1998). We used proper motions from Hipparcos or Jones \& Herbig (1979) to transform these coordinates to the epoch of the OVRO observations. Since the USNO-A2.0 coordinates do not resolve the binary systems, we assigned these coordinates to the photocenter of the emission measured in the HST images. The results are shown in Figure 1. In all cases, the source of the millimeter emission is unambiguous, since it aligns with the optical emission of a star to within 0 .'1-0.2. In each case, the stronger millimeter emission comes from the primary star, and in three of the four systems the secondary is undetected.

All of the millimeter detections, except for UX Tau A, are unresolved point sources. UX Tau A is marginally resolved; an elliptical Gaussian fit to the millimeter emission gives an FWHM size of 1 ". $1 \times 0$ ". 6 . As this is at the limit of our resolution, the exact size is uncertain; however, the clear difference between the source's integrated flux and peak flux indicates that it is definitely resolved. The components $\mathrm{HK}$ Tau A and B are consistent with unresolved point sources, but there is some additional diffuse emission between the two.

As can be seen in Table 2, our fluxes appear to be somewhat lower than the single-dish $1.3 \mathrm{~mm}$ fluxes for these sources. There are two possible explanations for this. One is that there is a systematic offset in the absolute flux calibration of the two data sets. As noted above, our absolute calibration is uncertain by $20 \%$; BSCG and Osterloh \& Beckwith (1995) use different calibration sources than we do and also cite a 
$20 \%$ flux calibration uncertainty. If we increase our fluxes by $20 \%$ and take into account the random uncertainties of each observation, then all of our measurements are still less than the single-dish fluxes, differing by 1.8, 0.9, 0.06, and 2.7 $\sigma$ for DK Tau, HK Tau, UX Tau, and V710 Tau, respectively. If we shift our data up by $20 \%$ and the single-dish fluxes down by $20 \%$, then the differences are $0.8,-0.4,-1.1$, and $1.6 \sigma$, with half of our measurements now being slightly higher than the single-dish fluxes.

Alternatively, if the flux calibrations are correct, then the discrepancy could be explained by the fact that the interferometric measurements are not sensitive to extended emission. Although a discrepancy between interferometric and single-dish flux measurements is expected for younger sources from which there is substantial envelope emission, $\mathrm{T}$ Tauri sources are expected to have little, if any, envelope component (see, e.g., HL Tau vs. L1551 IRS 5 in Lay et al. 1994). The percentage of the single-dish flux detected for our sources ranges from $46 \%$ to $83 \%$. Given the $u$ - $v$ coverage of our observations, emission must have a size scale greater than $5^{\prime \prime}$ before half of the emission is filtered out in the interferometric data.

Prato \& Simon (1997) have suggested that an extended circumbinary envelope could replenish the disks in T Tauri binary systems, although it is unclear how these envelopes could persist for so long (see, e.g., Clarke 2001). While our observations could be taken as support for existence of such an extended component, we caution that further observations are necessary to resolve the flux calibration issue.

\section{DISK PROPERTIES}

In this section, we attempt to determine the properties of the circumprimary and circumsecondary disks in our target systems. We first discuss what can be determined about the disks from existing optical and infrared data, and then we address the disk properties as revealed by our new observations.

\subsection{Disk Properties Inferred from Optical and Infrared Data}

Resolved spectra of the individual stars in our target binaries are presented in Cohen \& Kuhi (1979), Magazzù, Martín, \& Rebolo (1991), Hartigan et al. (1994), Monin et al. (1998), and Duchêne et al. (1999). We have quoted the range of literature spectral types in Table 1 . We adopted the spectral types given by Duchêne et al. (1999) for all systems except V710 Tau, for which we used the spectral types from Hartigan et al. (1994). In most cases, the different references agree fairly well about the spectral types; however, two cases merit some discussion. For DK Tau, Hartigan et al. (1994) list spectral types of $\mathrm{K} 7$ for both components, raising the question of whether it is clear which star is the primary. While Hartigan et al. (1994) do not show their spectra of DK Tau, the spectra shown in Monin et al. (1998) clearly show DK Tau B (their label) to be later in spectral type than DK Tau A. Second, optical and infrared photometry in the literature consistently show DK Tau A to be the brighter star. Thus, the choice of primary in this system seems clear.

In HK Tau, on the other hand, the spectra shown by Monin et al. (1998) appear to be quite similar in spectral type. We adopt the M2 and M3 classifications of Duchêne et al. (1999), which are based on the Monin et al. (1998) spectra, but we note that the two stars are extremely close in spectral type. HK Tau B is much fainter, but most (if not all) of this difference is due to its edge-on disk's blocking the direct starlight (Stapelfeldt et al. 1998; Koresko 1998).

The papers cited in the previous two paragraphs also present some resolved photometry, as do White \& Ghez (2001; $K$ and $L$ for all sources), Moneti \& Zinnecker (1991; $J H K$ for UX Tau), Koresko (1998; JHK for HK Tau), and Stapelfeldt et al. (1998; JHK, F606W, and F814W [HST filters similar to $V$ and $I$ ] for HK Tau).

All of these systems were detected by IRAS and thus have substantial mid- and far-infrared emission. However, the IRAS observations do not resolve the binaries, and thus it is unclear which of the binary components is the source of the infrared excess. Indeed, prior to the observations reported here, none of these systems had been resolved at a wavelength longer than $3.6 \mu \mathrm{m}$ ( $L$ band).

With the available resolved optical and infrared data, then, the best tracers of the presence of circumstellar material are $\mathrm{H} \alpha$ emission (presumed to arise from accretion onto the stellar surfaces) and $K-L$ color (which can reveal the presence of an infrared excess indicative of a disk). These quantities are given in Table 1 .

Among optical and near-infrared colors, $K-L$ is well suited for tracing circumstellar material, because it is relatively unaffected by interstellar reddening and because the photospheric colors of late-type stars have a relatively small range of values, minimizing the effect of uncertainties in spectral typing on the calculation of a color excess (White \& Ghez 2001). To determine the $K-L$ color excesses $\Delta(K-L)$ given in Table 1, we used the photospheric colors of Bessell $\&$ Brett (1988), which range from 0.10 to 0.20 for the range of spectral types in our sample.

Both the $\mathrm{H} \alpha$ emission and $\Delta(K-L)$ in Table 1 tell the same story. Stars classified as classical T Tauri stars (CTTSs) based on $\mathrm{H} \alpha$ equivalent width $(\geq 5 \AA$ for $\mathrm{K}$ stars, $\geq 10$ A for early M stars; see Martín 1997) also have significant $(>2.5-3 \sigma) \Delta(K-L)$ color excesses. The borderline CTTS V710 Tau B has only a borderline $\Delta(K-L)$ excess as well. All four systems thus have primary stars with evidence of disks. DK Tau and HK Tau also have secondaries with evidence of disks, while V710 B is marginal, and UX Tau B and $\mathrm{C}$ show little evidence of disk material.

\subsection{Disk Properties Inferred from Millimeter Observations}

As noted above, the most striking thing about the observed millimeter fluxes from these systems is how completely the primary stars dominate the systems' millimeter emission.

\subsubsection{Comparison with Optical and Infrared Disk Properties}

There is not a one-to-one correlation between the properties discussed in $\S 3.1$ and the millimeter fluxes in our observations (Fig. 2). While it appears that strong $\mathrm{H} \alpha$ emission and $K-L$ excess are necessary conditions for the presence of a millimeter detection, they are clearly not sufficient, since DK Tau B shows strong $\Delta(K-L)$ and $\mathrm{H} \alpha$, but no millimeter emission. This is most likely due to the fact that the optical and near-infrared emission arise in a relatively small region of the disk close to the star, while the millimeter flux is more broadly distributed. Thus, each diagnostic has its advantages. $\mathrm{H} \alpha$ emission and $K-L$ excess can be sensitive to relatively small disks that are undetected at millimeter wavelengths, while millimeter emission is a better tracer of global 


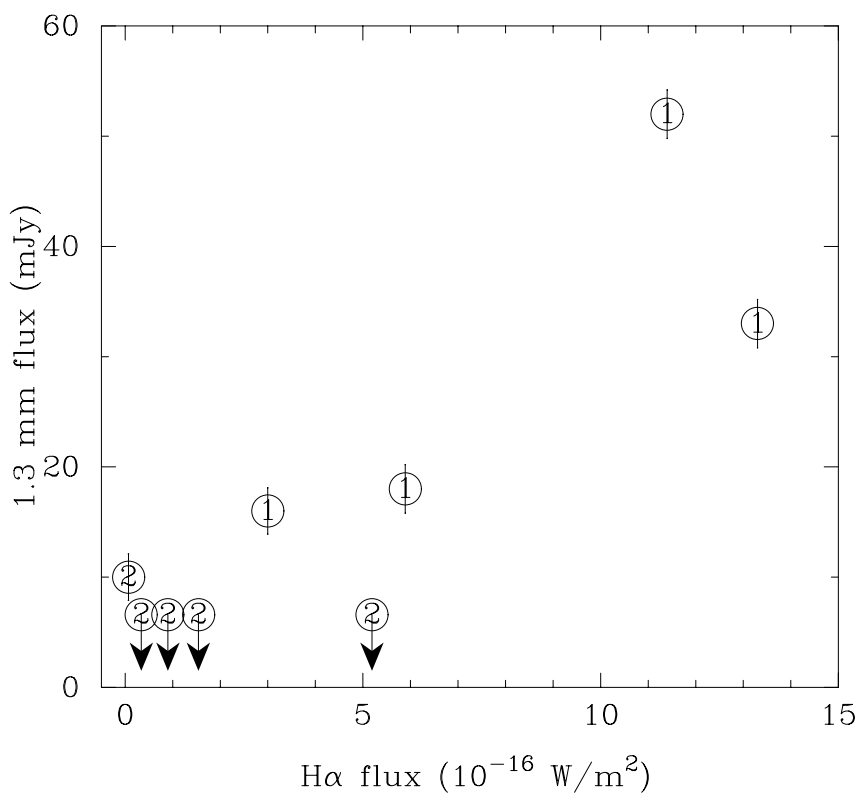

FIG. $2 a$

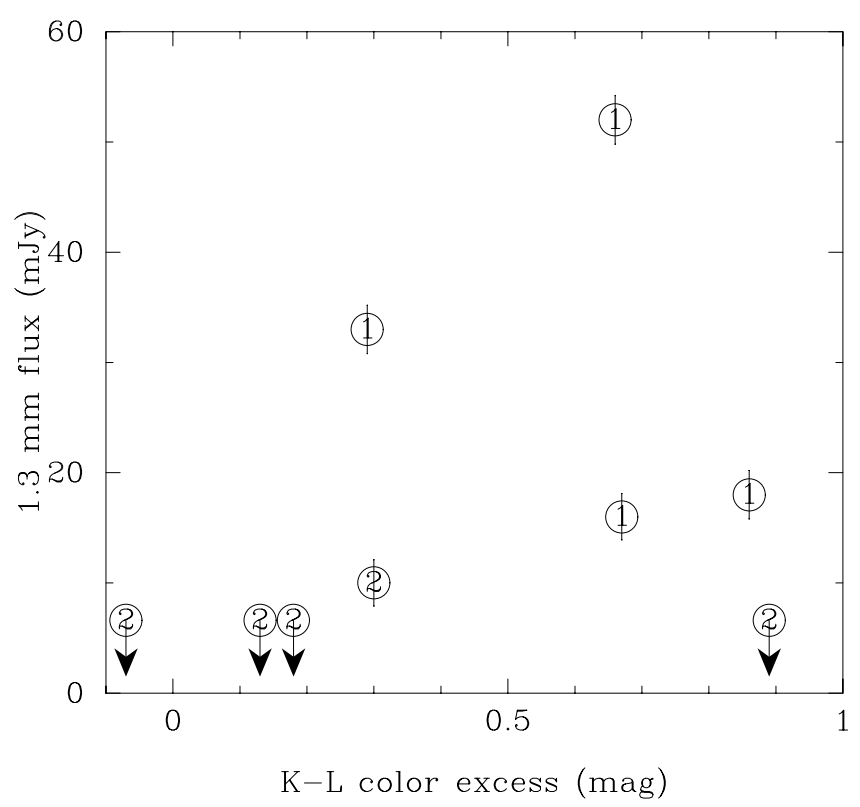

FIG. $2 b$

Fig. 2.- (a) Millimeter flux vs. H $\alpha$ emission-line flux. Symbols with " 1 " indicate primary stars, and those with " 2 " denote secondary or tertiary stars. There is no one-to-one correspondence between $\mathrm{H} \alpha$ and millimeter flux, indicating that disk accretion (traced by $\mathrm{H} \alpha$ ) is not strongly correlated with disk mass. (b) Millimeter flux vs. $K-L$ excess. Again, there is little correlation between $K-L$ excess, a tracer of inner disks, and the overall mass of the disk. $\mathrm{H} \alpha$ fluxes are derived from the equivalent widths in Table 1, and continuum fluxes are estimated from spectra in Monin, Ménard, \& Duchêne (1998) or $R$-band photometry (for V710 Tau only) in Hartigan et al. (1994). [See the electronic edition of the Journal for a color version of this figure.]

disk properties, especially disk mass. Disk mass is the property that is crucial for testing binary formation models, and it is there that we now turn our attention.

\subsubsection{Disk Mass Estimates}

Millimeter-wavelength observations have often been used in conjunction with optical and infrared data to model the spectral energy distributions of T Tauri stars, with the optical and infrared data providing some constraint on the disk temperature distribution and the millimeter data providing a tracer of optically thin material in order to determine the disk mass (see, e.g., BSCG; Osterloh \& Beckwith 1995). However, there is not enough resolved mid- and far-infrared data available for these binaries to justify detailed modeling of their spectral energy distributions.

Limits on the disk masses can be estimated by assuming that all emission is from optically thin material with a given temperature. These estimated disk masses (Table 3) are a lower limit to the true disk mass. We use the canonical value for the dust emissivity $\kappa_{\nu}$ from Hildebrand (1983) of $0.1 \mathrm{~cm}^{2}$ $\mathrm{g}^{-1}$ at $\lambda=250 \mu \mathrm{m}$, with a frequency scaling $\kappa_{\nu} \propto \nu^{\beta}$ of $\beta=1$.

The assumption that the disks are entirely optically thin at $1.3 \mathrm{~mm}$ is unlikely to be correct for the primary stars with detected $1.3 \mathrm{~mm}$ flux, and so these masses are lower limits to the true disk mass. However, we note that these mass estimates are comparable to those obtained from detailed models (BSCG; Osterloh \& Beckwith 1995) of stars with similar millimeter fluxes, suggesting that the optically thin approximation may be reasonably good. In their $\mathrm{T}$ Tauri disk survey at $\lambda=2.7 \mathrm{~mm}$, Dutrey et al. (1996) estimated that optically thick emission accounted for $\lesssim 10 \%$ of the total mass for 13 of 15 sources. Using their assumed disk model to extend these results to $\lambda=1.3 \mathrm{~mm}$, optically thick emission at $\lambda=1.3 \mathrm{~mm}$ accounts for less than $20 \%$ of the total mass in 13 of 15 sources.

\subsubsection{Disk Size Estimates}

Alternatively, if we assume that the millimeter flux comes entirely from optically thick material, we can estimate the minimum size of the emitting region. If a disk inclination value is assumed (we used $\cos i=0.5$ ) and the temperature is described as a power-law function of radius, $T(r)=T\left(r_{0}\right)\left(r / r_{0}\right)^{-q}$, then for a given flux, the outer radius for a completely optically thick disk depends only on $T\left(r_{0}\right)$ and $q$. For each source, we calculated the outer radius using $T\left(r_{0}\right)=150 \mathrm{~K}$ and $q=0.5$. The calculated radii are given in Table 3. Calculations using the $T\left(r_{0}\right)$ and $q$-values derived for each source in BSCG yielded similar results. These radii can be interpreted as lower limits to the true disk radii, since it is likely that some of the emission is optically thin. The

TABLE 3

Disk Mass AND RADIUS Lower Limits

\begin{tabular}{|c|c|c|c|}
\hline \multirow[b]{2}{*}{ SOURCE } & \multicolumn{2}{|c|}{$\begin{array}{l}\text { Optically Thin DisK Mass } \\
\qquad\left(\times 10^{-3} M_{\odot}\right)\end{array}$} & \multirow{2}{*}{$\begin{array}{c}\text { Optically THick } \\
\text { Disk RadiUs } \\
\text { (AU) }\end{array}$} \\
\hline & $T=15 \mathrm{~K}$ & $T=30 \mathrm{~K}$ & \\
\hline DK Tau A.............. & 3.4 & 1.7 & 9.1 \\
\hline DK Tau B ............. & $<1.2$ & $<0.6$ & $\ldots$ \\
\hline HK Tau A.............. & 3.0 & 1.5 & 8.4 \\
\hline HK Tau B .............. & 1.9 & 0.9 & 6.1 \\
\hline UX Tau A ................. & 9.7 & 4.9 & 18.5 \\
\hline UX Tau B .............. & $<1.2$ & $<0.6$ & $\ldots$ \\
\hline UX Tau C .............. & $<1.2$ & $<0.6$ & $\ldots$ \\
\hline V710 Tau A............ & 6.2 & 3.1 & 13.6 \\
\hline V710 Tau B ........... & $<1.2$ & $<0.6$ & $\ldots$ \\
\hline
\end{tabular}


derived radii are similar to the limits found by Dutrey et al. (1996) for circumstellar disks around single T Tauri stars.

\section{DISCUSSION}

The $1.3 \mathrm{~mm}$ flux in these systems is clearly dominated by the primary star. The essential question for comparison of these observations with predictions of theories of binary formation is whether or not this larger flux indicates a larger disk mass around the primaries as well.

While some of the observed flux difference may be attributable to the primaries' disks being hotter, we argue that the primaries must in fact have more massive disks than the secondaries. The primary and secondary stellar effective temperatures differ by less than $10 \%$ in three of the four systems, and by $22 \%-28 \%$ in UX Tau. A temperature difference of this magnitude is not sufficient to account for the observed flux difference if the disks have similar masses and opacities.

Another argument that could be made against the higher millimeter fluxes resulting from higher mass disks is that the disk inclinations of the secondaries might be different from those of the primaries, with the primaries presenting a larger projected surface area and thus a larger millimeter flux from (perhaps) a similar disk mass. The problem with this hypothesis is that it requires a coincidence, namely, that secondaries happen to be more edge-on to Earth than the primaries in all four cases. Another problem arises in the case of HK Tau, where the inclination of the secondary disk is known to be fairly edge-on to Earth (roughly $5^{\circ}$; Stapelfeldt et al. 1998) and the primary and secondary disks are clearly not coplanar. This is the system that has perhaps the most edge-on secondary disk, yet it is the only system in our sample in which the secondary was detected. This argues strongly against all the secondary disks being undetected because of an edge-on geometry. For equal-mass, optically thin primary and secondary disks, only extreme inclination angles for the secondaries would result in the primary/ secondary flux ratios measured here. Inclination angles this extreme would probably obscure the optical emission from the secondary, which is only the case for HK Tau (Fig. 1). Finally, Jensen, Donar, \& Mathieu (2000) find that among wide binaries in general, primary and secondary disks tend to be aligned with each other to within $\sim 20^{\circ}$, making it unlikely that we would see significant flux differences due to inclination differences alone.

Thus, the secondaries not only have lower millimeter fluxes, but they almost certainly have lower mass disks. This is in excellent agreement with binary formation models of scale-free fragmentation (see, e.g., Bate 2000; Clarke 2001).

Examining the relationship between stellar mass and disk mass suggests that the disk properties we observe are somehow related to the dynamics of binary formation and evolution and not just to the mass of each individual star. Some of the secondary stars in the systems discussed here are roughly equal in mass to some of the primaries; for example, HK Tau A and DK Tau B are both spectral type M1 and therefore are very close in mass; V710 Tau A is also close at spectral type M0.5. Despite this similarity, HK Tau A and V710 Tau A, the more massive stars in their respective systems, have detectable millimeter flux (and therefore more massive disks), while DK Tau B has a very small millimeter flux, below our sensitivity limit (Fig. 3). While our sample is small and thus we cannot draw definitive conclusions about all young binaries, our data suggest that it is not the individual stellar masses in a binary system that determine the distribution of disk mass within the system. Rather, the primary (regardless of its absolute mass) retains a more substantial disk. In this scenario, the fact that both components in HK Tau have detectable disks is consistent with the fact that it is the only system in which the primary and secondary spectral types are virtually indistinguishable.

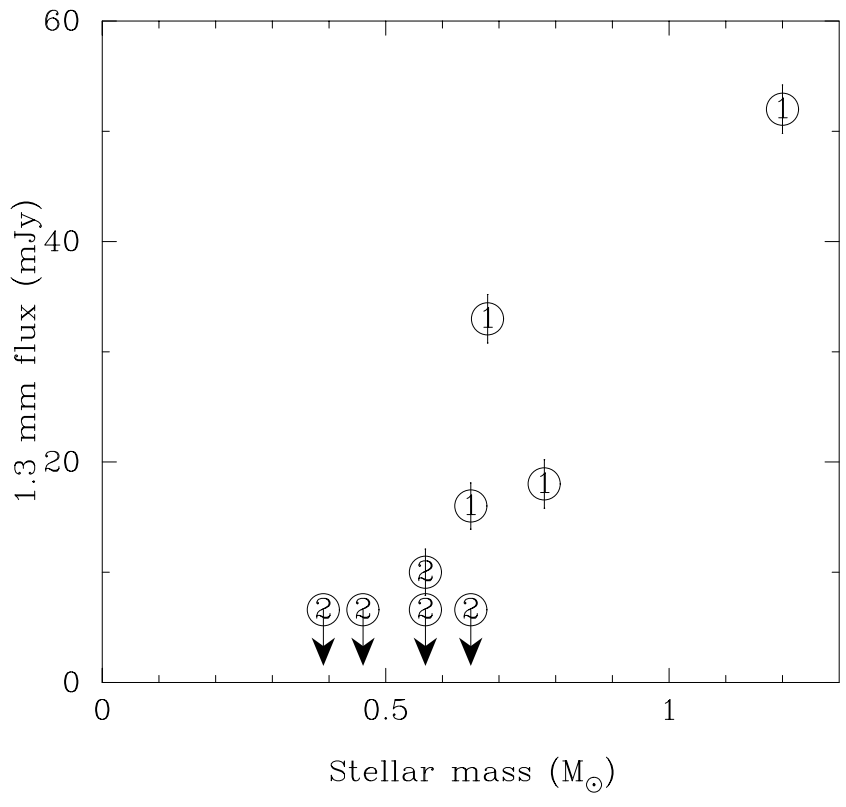

FIG. $3 a$

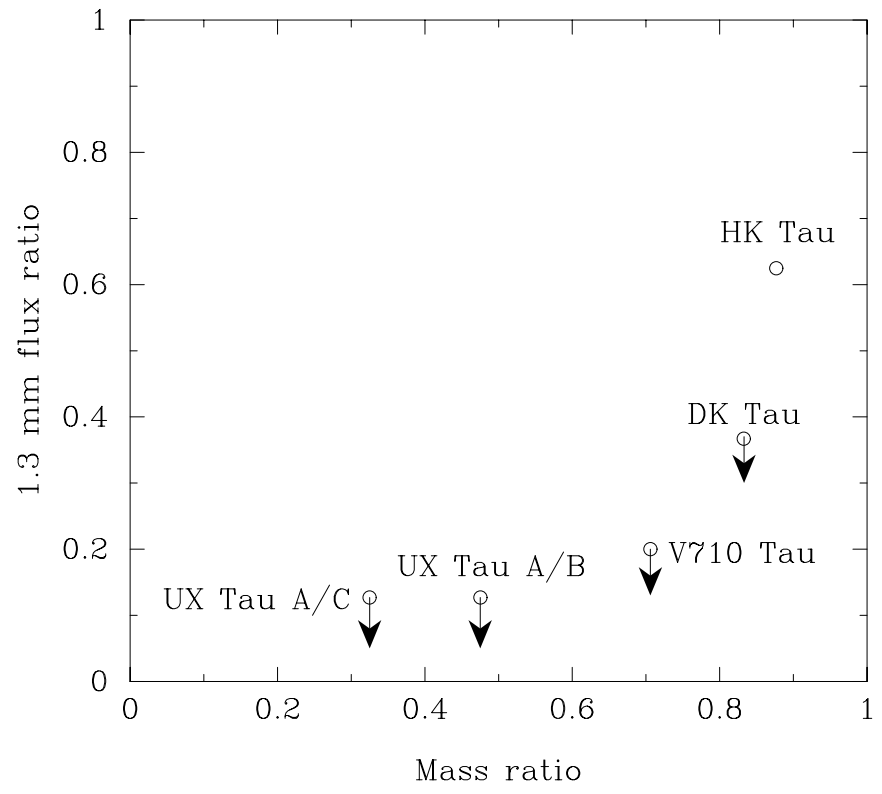

FIG. $3 b$

FIG. 3.- (a) Millimeter flux vs. stellar mass; symbols are as in Fig. 2. There is overlap between the primary and secondary mass distributions, with all primaries being detected while most secondaries, even those with masses comparable to some primaries, are undetected. (b) Flux ratio from our millimeter observations vs. the stellar mass ratio. Only when the stellar mass ratio is very close to one is the secondary detected. Both panels suggest that the star's relative mass within the binary system is more important for its disk properties than its absolute mass. [See the electronic edition of the Journal for a color version of Fig. 3a.] 
It is also notable that the disk masses derived for the primaries are similar to those derived for single $\mathrm{T}$ Tauri stars by BSCG and Osterloh \& Beckwith (1995), especially considering that our mass estimates are lower limits. This suggests that a wide ( $>200 \mathrm{AU}$ ) binary companion does not prevent the formation of a circumstellar disk that is near the mass of the minimum mass solar nebula, consistent with the conclusions of Jensen et al. (1996b). In fact, this result strengthens the prospects for planet formation in wide binaries, since it suggests that most of the disk mass in these systems resides in a single, more massive disk rather than two smaller disks, thus providing a larger reservoir of material for planet formation around the primary.

The upper limits on the millimeter flux from the secondaries do not, in and of themselves, set these stars apart from single stars; roughly half of the single stars in the sample of Osterloh \& Beckwith (1995) were not detected at $1.3 \mathrm{~mm}$. What is striking, as emphasized above, is the comparison between the millimeter emission of the primary and that of the secondary. This is especially notable since a comparison of primary and secondary disks within a given binary system is effectively controlled for the effects of age and formation environment. These factors allow for the persistence of a substantial disk around the primaries in our sample, and yet most of the secondaries are relatively devoid of disk material.

Several studies (see, e.g., the review by Prato \& Monin 2001) have shown that $T$ Tauri binaries tend to occur in matched pairs; i.e., it is much more common to find CTTSs paired with other CTTSs and weak-lined T Tauri stars (WTTSs) paired with other WTTSs than it is to find mixed CTTS/WTTS pairs. The fact that both stars in a system are CTTSs does not mean that their disks are similar, however. While $\mathrm{H} \alpha$ traces disk accretion, it is not a good tracer of disk mass (see, e.g., BSCG). Our results here reinforce the disparity between accretion diagnostics and disk mass: while most of the secondaries are CTTSs, they have very low mass disks (Table 3), and thus their disks are fairly different from those around the primaries. The most notable example is DK Tau B, with an $\mathrm{H} \alpha$ emission equivalent width of $118 \AA$ but no detectable $\lambda=1.3 \mathrm{~mm}$ emission. Thus, while both primary and secondary in a binary system are often CTTSs, primaries and secondaries can have very different disk masses.

\section{CONCLUSIONS}

We have presented $\lambda=1.3 \mathrm{~mm}$ continuum images of four young binary systems, showing that the primary star has a more massive disk in all cases; this is consistent with leading models of binary formation by fragmentation. These circumprimary disks are comparable in mass to those found around single T Tauri stars, indicating that the presence of a wide binary formation does not prevent the formation and survival of a disk massive enough to form a solar system like our own. In some systems, the secondary star shows evidence of strong accretion, but no detectable millimeter emission, indicating that the reservoir of accreting material has a relatively low mass. None of the systems observed has detectable ${ }^{13} \mathrm{CO}(2 \rightarrow 1)$ emission at the sensitivity limit of our observations.

This work was performed in part at the Interferometry Science Center, California Institute of Technology. The Owens Valley Millimeter Array is supported by NSF grant AST 96-13717. E. L. N. J. gratefully acknowledges the support of the National Science Foundation's Life in Extreme Environments program, through grant AST 99-96278, and Swarthmore College, through a James Michener Fellowship. We thank the anonymous referee, whose detailed comments improved the presentation of this work. We thank Rabi Whitaker for a careful reading of the paper.
Akeson, R. L., Koerner, D. W., \& Jensen, E. L. N. 1998, ApJ, 505, 358

Baggett, S., et al. 2002, HST WFPC2 Data Handbook, Version 4.0, ed. B. Mobasher (Baltimore: STScI)

Baraffe, I., Chabrier, G., Allard, F., \& Hauschildt, P. H. 1998, A\&A, 337, 403

Bate, M. R. 2000, MNRAS, 314, 33

Beckwith, S. V. W., Sargent, A. I., Chini, R., \& Güsten, R. 1990, AJ, 99, 924 (BSCG)

Bessell, M. S., \& Brett, J. M. 1988, PASP, 100, 1134

Clarke, C. J. 2001, in IAU Symp. 200, Birth and Evolution of Binary Stars, ed. H. Zinnecker \& R. D. Mathieu (San Francisco: ASP), 346

Cohen, M., \& Kuhi, L. V. 1979, ApJS, 41, 743

Cox, A. N., ed. 2000, Allen's Astrophysical Quantities (4th ed.; New York: AIP)

Duchêne, G., Monin, J.-L., Bouvier, J., \& Ménard, F. 1999, A\&A, 351, 954

Dutrey, A., Guilloteau, S., Duvert, G., Prato, L., Simon, M., Schuster, K., \& Ménard, F. 1996, A\&A, 309, 493

Duvert, G., Dutrey, A., Guilloteau, S., Ménard, F., Schuster, K., Prato, L., \& Simon, M. 1998, A\&A, 332, 867

Ghez, A. M. 2001, in IAU Symp. 200, Birth and Evolution of Binary Stars, ed. H. Zinnecker \& R. D. Mathieu (San Francisco: ASP), 210

Guilloteau, S., Dutrey, A., \& Simon, M. 1999, A\&A, 348, 570

Hartigan, P., Strom, K. M., \& Strom, S. E. 1994, ApJ, 427, 961

Hildebrand, R. H. 1983, QJRAS, 24, 267

Jensen, E. L. N., Donar, A. X., \& Mathieu, R. D. 2000, in IAU Symp. 200

Poster Proceedings, Birth and Evolution of Binary Stars, ed. B. Reipurth

\& H. Zinnecker (Potsdam: Astrophys. Inst.), 85

Jensen, E. L. N., Koerner, D. W., \& Mathieu, R. D. 1996a, AJ, 111, 2431

\section{RFERENCES}

Jensen, E. L. N., Mathieu, R. D., \& Fuller, G. A. 1996b, ApJ, 458, 312

Jones, B. F., \& Herbig, G. H. 1979, AJ, 84, 1872

Koerner, D. W., Sargent, A. I., \& Beckwith, S. V. W. 1993, ApJ, 408, L93

Koresko, C. D. 1998 , ApJ, 507, L145

Lay, O. P., Carlstrom, J. E., Hills, R. E., \& Phillips, T. G. 1994, ApJ, 434, L75

Leinert, C., Zinnecker, H., Weitzel, N., Christou, J., Ridgway, S. T., Jameson, R., Haas, M., \& Lenzen, R. 1993, A\&A, 278, 129

Luhman, K. L. 1999, ApJ, 525, 466 2000, ApJ, 544, 1044

Magazzù, A., Martín, E. L., \& Rebolo, R. 1991, A\&A, 249, 149

Martín, E. L. 1997, A\&A, 321, 492

Mathieu, R. D. 1994, ARA\&A, 32, 465

Mathieu, R. D., Ghez, A. M., Jensen, E. L. N., \& Simon, M. 2000, in Protostars and Planets IV, ed. V. Mannings, A. P. Boss, \& S. S. Russell (Tucson: Univ. Arizona Press), 703

Monet, D., et al. 1998, The USNO-A2.0 Catalogue (Washington: USNO)

Moneti, A., \& Zinnecker, H. 1991, A\&A, 242, 428

Monin, J.-L., Ménard, F., \& Duchêne, G. 1998, A\&A, 339, 113

Osterloh, M. \& Beckwith, S. V. W. 1995, ApJ, 439, 288

Prato, L., \& Monin, J.-L. 2001, in IAU Symp. 200, Birth and Evolution of Binary Stars, ed. H. Zinnecker \& R. D. Mathieu (San Francisco: ASP), 313

Prato, L., \& Simon, M. 1997, ApJ, 474, 455

Stapelfeldt, K. R., Krist, J. E., Ménard, F., Bouvier, J., Padgett, D. L., \& Burrows, C. J. 1998, ApJ, 502, L65

White, R. J., \& Ghez, A. M. 2001, ApJ, 556, 265 